\title{
High-Entropy 2D Carbide MXenes
}

\author{
Srinivasa Kartik Nemani ${ }^{1,2}$, Bowen Zhang ${ }^{1,2,3}$, Brian C. Wyatt ${ }^{1,2}$, Zachary D. Hood ${ }^{4}$, Sukriti \\ Manna $^{5}$, Rasoul Khaledialidusti ${ }^{6}$, Weichen Hong ${ }^{1}$, Michael G. Sternberg ${ }^{5}$, Subramanian \\ Sankaranarayanan ${ }^{5,7, *}$, Babak Anasori ${ }^{1,2, *}$
}

1. Department of Mechanical and Energy Engineering, Purdue School of Engineering and Technology, Indiana University-Purdue University Indianapolis, Indianapolis, Indiana, 46202, United States

2. Integrated Nanosystems Development Institute, Indiana University-Purdue University Indianapolis, Indianapolis, Indiana, 46202, United States

3. Henan Key Laboratory of Photovoltaic Materials, Henan University, Kaifeng 475004, P. R. China

4. Applied Materials Division, Argonne National Laboratory, Lemont, Illinois 60439, United States

5. Center for Nanoscale Materials, Argonne National Laboratory, Lemont, Illinois 60439, United States

6. Department of Mechanical and Industrial Engineering, Norwegian University of Science and Technology (NTNU), 7491 Trondheim, Norway

7. Department of Mechanical and Industrial Engineering, University of Illinois, Chicago, Illinois 60607, United States

*Address correspondence to: skrssank@uic.edu,banasori@iupui.edu

\begin{abstract}
Two-dimensional (2D) transition metal carbides and nitrides, known as MXenes, are a fastgrowing family of 2D materials. MXenes 2D flakes have $n+1(n=1-4)$ atomic layers of transition metals interleaved by carbon/nitrogen layers, but to-date remain limited in composition to one or two transition metals. In this study, through the use of four transition metals, we report the synthesis of multi-principal element high-entropy $\mathrm{M}_{4} \mathrm{C}_{3} \mathrm{~T}_{x}$ MXenes. Specifically, we introduce two high-entropy MXenes, $\mathrm{TiVNbMoC}_{3} \mathrm{~T}_{x}$ and $\mathrm{TiVCrMoC}_{3} \mathrm{~T}_{x}$, as well as their precursor $\mathrm{TiVNbMoAlC}_{3}$ and $\mathrm{TiVCrMoAlC}_{3}$ high-entropy MAX phases. We used a combination of real and reciprocal space characterization (x-ray diffraction, x-ray photoelectron spectroscopy, energy dispersive $\mathrm{x}$-ray spectroscopy, and scanning transmission electron microscopy) to establish the structure, phase purity, and equimolar distribution of the four transition metals in high-entropy MAX and MXene phases. We use first-principles calculations to compute the formation energies and explore synthesizability of these high-entropy MAX phases. We also show that when three transition metals are used instead of four, under similar synthesis conditions to those of the fourelement MAX phase, two different MAX phases can be formed (i.e. no pure single-phase forms). This finding indicates the importance of configurational entropy in stabilizing the desired singlephase high-entropy MAX over multiphases of MAX, which is essential for the synthesis of phasepure high-entropy MXenes. The synthesis of high-entropy MXenes significantly expand the compositional variety of the MXene family to further tune their properties, including electronic, magnetic, electrochemical, catalytic, high temperature stability, and mechanical properties.
\end{abstract}

Keywords: MXenes, 2D materials, Multi-principal elements, high-entropy, transition metal, carbides 
MXenes are a large family of two-dimensional (2D) transition metal carbides and nitrides, which were first synthesized in 2011. ${ }^{1,2}$ MXenes have a chemical formula of $\mathrm{M}_{n+1} \mathrm{X}_{n} \mathrm{~T}_{x}(n=1-$ 4), where a 2D flake is comprised of $n+1$ atomic layers of transition metals from groups 3 to 6 of the periodic table interleaved by a layer of carbon or nitrogen $(\mathrm{X})$ between the M layers. ${ }^{3,4} \mathrm{In}$ addition, the outer transition metal atomic layers are bonded to surface terminations, such as - $\mathrm{O}$, $-\mathrm{F}$, and $-\mathrm{OH}^{5}$ In the past decade, more than thirty MXene compositions have been synthesized while many more have been studied by computational methods. ${ }^{6,7}$ MXene synthesized to date have exhibited metallic electrical conductivity (up to $20,000 \mathrm{~S} / \mathrm{cm}$ for $\mathrm{Ti}_{3} \mathrm{C}_{2} \mathrm{~T}_{x}$ ), ${ }^{8}$ high in-plane mechanical stiffness (up to $386 \pm 13 \mathrm{GPa}$ for $\mathrm{Nb}_{4} \mathrm{C}_{3} \mathrm{~T}_{x}$ ), ${ }^{9}$ and impressive catalytic as well as electrochemically active behavior. ${ }^{3,}{ }^{10}$ MXenes are generally synthesized by selective etching of the A-group elements (mostly group 13-16 of the periodic table) from their carbide and nitride precursors, which are mostly MAX phases. ${ }^{11}$

MXenes with two transition metals (known as double transition metal (DTM) MXenes) are becoming more prominent in this 2D family since 2015. ${ }^{12-15}$ DTM MXenes provide a limitless range of possible compositions of MXenes in the form of random solid solutions of transition metals, where two transition metals randomly occupy the different $M$ layers, which provides control and tunability of MXenes' properties. ${ }^{15-17}$ In addition to adding a second transition metal in previously seen structures of MXene, DTM MXenes also provide novel structures which are not seen in mono-transition metal MXenes, such as out-of-plane ordered $\mathrm{M}_{3} \mathrm{C}_{2} \mathrm{~T}_{x}$ and $\mathrm{M}_{4} \mathrm{C}_{3} \mathrm{~T}_{x}{ }^{12}$ or in-plane ordered $\mathrm{M}_{2} \mathrm{CT}_{x},{ }^{13}$ and a new MXene structure of $\mathrm{M}_{5} \mathrm{C}_{4} \mathrm{~T}_{x}$ solid solution. ${ }^{4} \mathrm{DTM}$ MXenes have opened new avenues for application specific compositional tailoring which are otherwise impossible in 2D nanomaterials or mono-transition metal MXenes, such as semiconductive, magnetic, or to be topological materials in out-of-plane $\mathrm{M}_{3} \mathrm{C}_{2} \mathrm{~T}_{x}$ and $\mathrm{M}_{4} \mathrm{C}_{3} \mathrm{~T}_{x}$ MXenes. ${ }^{16,18,19}$

Since 2004, multi-principal element (MPE) high-entropy alloys in bulk 3D crystalline compounds have emerged as promising materials. ${ }^{20-24}$ The high-entropy metal alloy concept is a materials synthesis strategy where several (usually five or more) elements are combined in high and near equiatomic concentrations ( $5-35$ at.\%) to stabilize a single-phase formation, instead of multiphases of solid solutions with different compositions and crystal structures. ${ }^{24}$ They present a vast compositional space with resulting structures and properties that are not observed in single- 
phase or traditional alloys with only one or two principal elements. ${ }^{24}$ The high-entropy alloy concept has been most notably applied to transition metal bulk alloy systems, where exceptional combinations of mechanical properties, thermal stability, and oxidation resistance have been reported. Since 2015, the high-entropy concept has expanded further to 3D crystalline ceramics, where four or more metal elements in equimolar or near equimolar proportions form high-entropy structures with non-metal elements such as $\mathrm{O}, \mathrm{C}, \mathrm{N}$, and B. ${ }^{25-32}$ Some of the bulk 3D crystalline high-entropy transition metal carbides properties surpass those of their mono-transition metal carbide, such as improved hardness, oxidation resistance, wear resistance and low thermal conductivity ${ }^{25,27,33-42}$ This has led to their application in thermal, corrosive, extreme temperature and pressure environments. ${ }^{29,30}$ However, the field of high-entropy transition metal carbides is in its nascent stages of exploration with only a few studies being reported to date. Unlike the extensive number of studies on high-entropy 3D crystalline solids, very little has been reported on high-entropy 2D materials. While a few studies explored high-entropy MPE 2D transition metal dichalcogenides, ${ }^{43,44}$ to the best of our knowledge, there are currently no reports on high-entropy 2D carbides.

Inspired by the two fast-growing fields of high-entropy compounds and 2D MXenes, we report on successful synthesis of two high-entropy MXenes in this study. To do so, we first synthesized MXene precursors of layered MPE MAX carbides, $\mathrm{TiVNbMoAlC}_{3}$ and TiVCrMoAlC $_{3}$ phases (Figure 1a, b). These MAX phases are two new MPE phases in the potentially large family of high-entropy MAX compositions. After we confirmed the single-phase purity of the high-entropy MAX phases, we exfoliated and delaminated these MAX phases to synthesize $\mathrm{TiVNbMoC}_{3} \mathrm{~T}_{x}$ and $\mathrm{TiVCrMoC}_{3} \mathrm{~T}_{x}$ (Figure 1c, d) $2 \mathrm{D}$ MXenes. In all these synthesized high-entropy phases, the transition metal stoichiometric ratios were retained at 1:1:1:1 $( \pm 0.2)$ for $M^{1}: M^{2}: M^{3}: M^{4}$. We theoretically verify their synthesizability by calculating the enthalpy of formation for these MAX phase systems using density functional theory (DFT) and a spinpolarized version of the generalized gradient approximation (GGA) ${ }^{45}, 46$ This development of layered high-entropy MPE MAX phases and their high-entropy MPE MXenes adds a new exotic family of MXenes to this large and fast-growing field and provides a new method of designing 2D materials with tailored and tunable material properties. 


\section{RESULTS AND DISCUSSION}

The synthesis of MXenes is a top-down process, which requires to make their precursor MAX phases. We synthesized the high-entropy MAX phases by reactive sintering of elemental powders (Figure 1a). Details of the full experimental approach can be found in the Methods Section. Briefly, we first mixed an equimolar ratio of four transition metals $\mathrm{M}^{1}, \mathrm{M}^{2}, \mathrm{M}^{3}$, and $\mathrm{M}^{4}$ with $\mathrm{Al}$ and $\mathrm{C}$ $\mathrm{M}^{1}: \mathrm{M}^{2}: \mathrm{M}^{3}: \mathrm{M}^{4}: \mathrm{Al}: \mathrm{C}$ in 1:1:1:1:1.1:2.7 stoichiometric ratio to obtain $\mathrm{TiVNbMoAlC}_{3}$ and $\mathrm{TiVCrMoAlC}_{3} \mathrm{MAX}$ phases (Figure 1b). We chose our initial starting powders of a combination of transition metals based on their existence established as stable solid solutions in MAX and MXene phases in previous theoretical and experimental studies. 1, 12, 15, 16, 47, 48 After reactive sintering at $1600{ }^{\circ} \mathrm{C}$ for four hours under argon atmosphere (Figure 1a), the sintered blocks were milled to obtain fine powders.

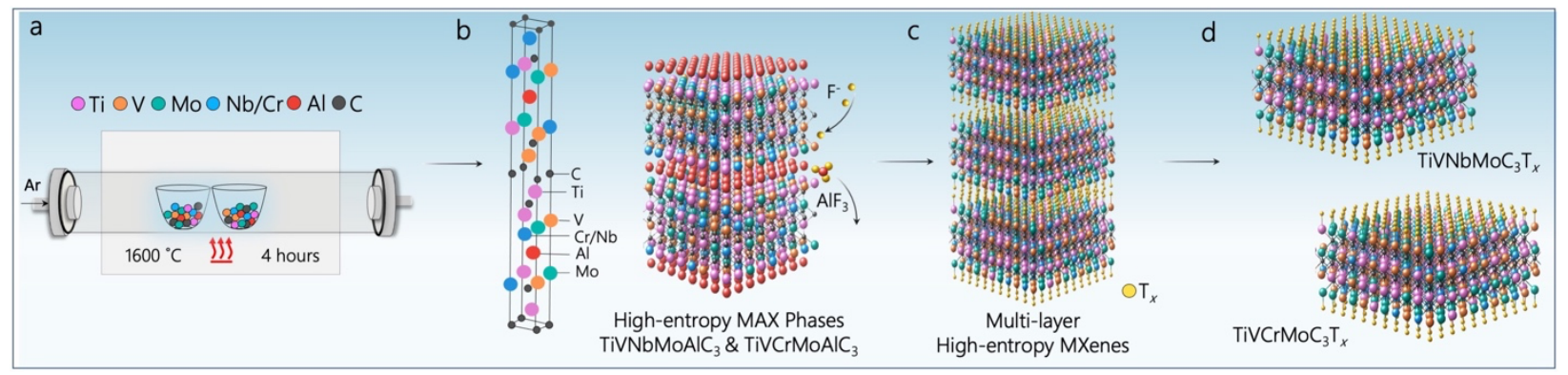

Figure 1. Schematic for synthesis of high-entropy MAX and MXenes. (a) Reactive sintering of high-entropy MAX phases. Elemental powder mixtures with stoichiometric molar ratios were sintered in alumina crucibles inside a tube furnace. (b) A MAX unit cell (left) of $\mathrm{M}^{1} \mathrm{M}^{2} \mathrm{M}^{3} \mathrm{M}^{4} \mathrm{AlC}_{3}$ with elements Ti (pink), $\mathrm{V}$ (orange), $\mathrm{Nb}$ or $\mathrm{Cr}$ (blue), Mo (green), $\mathrm{Al}$ (red), and C (black). The synthesized MAX phasea with layered transition metal layers are comprised of four transition metal elements, with aluminum (red) and carbon (black) atomic layers in a $\mathrm{M}_{4} \mathrm{AlC}_{3} \mathrm{MAX}$ structure (right). (c) Selective etching of the Al layer by hydrofluoric acid to synthesize multi-layer high-entropy MXenes. The surface terminations on the basal planes of MXenes $\left(T_{x}\right)$ are shown in yellow. (d) Delamination of multi-layer MXenes is completed via organic molecule intercalants, which leads to formation of single flakes of high-entropy MXenes $\mathrm{TiVNbMoC}_{3} \mathrm{~T}_{x}$ and $\mathrm{TiVCrMoC}_{3} \mathrm{~T}_{x}$.

First, we used x-ray diffraction (XRD) on the two sintered powders of Ti:V:Nb:Mo:Al:C and Ti:V:Cr:Mo:Al:C and analyze their spectra to verify the formation of $\mathrm{TiNNbMoAlC}_{3}$ and $\mathrm{TiVCrMoAlC}_{3}$ MAX phases (bottom patterns in Figure 2a and b). Using these spectra, we confirmed the presence of $\mathrm{M}_{4} \mathrm{AlC}_{3} \mathrm{MAX}$ phase peaks. Through identification of their characteristic $(00 l)$ and in-plane peaks, we calculated their $a$-lattice parameters ( $a$-LPs) to be 3.038 and $2.97 \AA$ and $c$-LPs of 23.590 and $22.978 \AA$ for $\mathrm{TiVNbMoAlC}_{3}$ and $\mathrm{TiVCrMoAlC}_{3}$, respectively. The identified peaks and lattice parameters of the formed $\mathrm{TiVNbMoAlC}_{3}$ and $\mathrm{TiVCrMoAlC}_{3} \mathrm{MAX}$ phases are in good agreement with previously reported $\mathrm{M}_{4} \mathrm{AlC}_{3}$ phases. ${ }^{48,49}$ After identification of 
the characteristic $\mathrm{M}_{4} \mathrm{AlC}_{3}$ diffraction peaks, we focused on evaluating the presence of impurity phases. In this analysis, we identified that common impurities in the synthesis of these MAX phases include very small amounts of Al-Mo and Mo-V intermetallic phases in both $\mathrm{TiVNbMoAlC}_{3}$ and $\mathrm{TiVCrMoAlC}_{3}$. The identification of very small intermetallic impurities in our system with no other clearly identifiable carbide or oxide impurities via XRD analysis indicated that our equimolar high-entropy stabilization synthesis method forms highly pure $\mathrm{M}_{4} \mathrm{AlC}_{3} \mathrm{MAX}$ phases. This is important for the subsequent selective etching and delamination of these phases to 2D MXene flakes.

After confirmation of the formation of targeted $\mathrm{M}_{4} \mathrm{AlC}_{3} \mathrm{MAX}$ phases with XRD, we next focused on characterization of the resultant composition of the formed phases using energydispersive $\mathrm{x}$-ray spectroscopy (EDS) combined with scanning electron microscopy (SEM). To distinguish the compositions in the resulting MAX phases, we conducted EDS point scans on several MAX particles shown in the SEM micrographs (Figure 3a and b). The average EDS results for the four transition metals in each composition are shown in Table $\mathbf{S 1}$ for $\mathrm{TiVNbMoAlC}_{3}$ and $\mathrm{TiVCrMoAlC}_{3}$. These results indicate an equimolar ratio of the four transition metals as Ti:V:Nb:Mo $\quad 0.9 \pm 0.2: 1.1 \pm 0.2: 1.1 \pm 0.2: 0.9 \pm 0.3 \quad$ and Ti:V:Cr:Mo ratio of $1.0 \pm 0.1: 1.1 \pm 0.1: 1.0 \pm 0.1: 0.9 \pm 0.1$. This is the first confirmation that we have successfully synthesized two MPE MAX phases. ${ }^{8}$
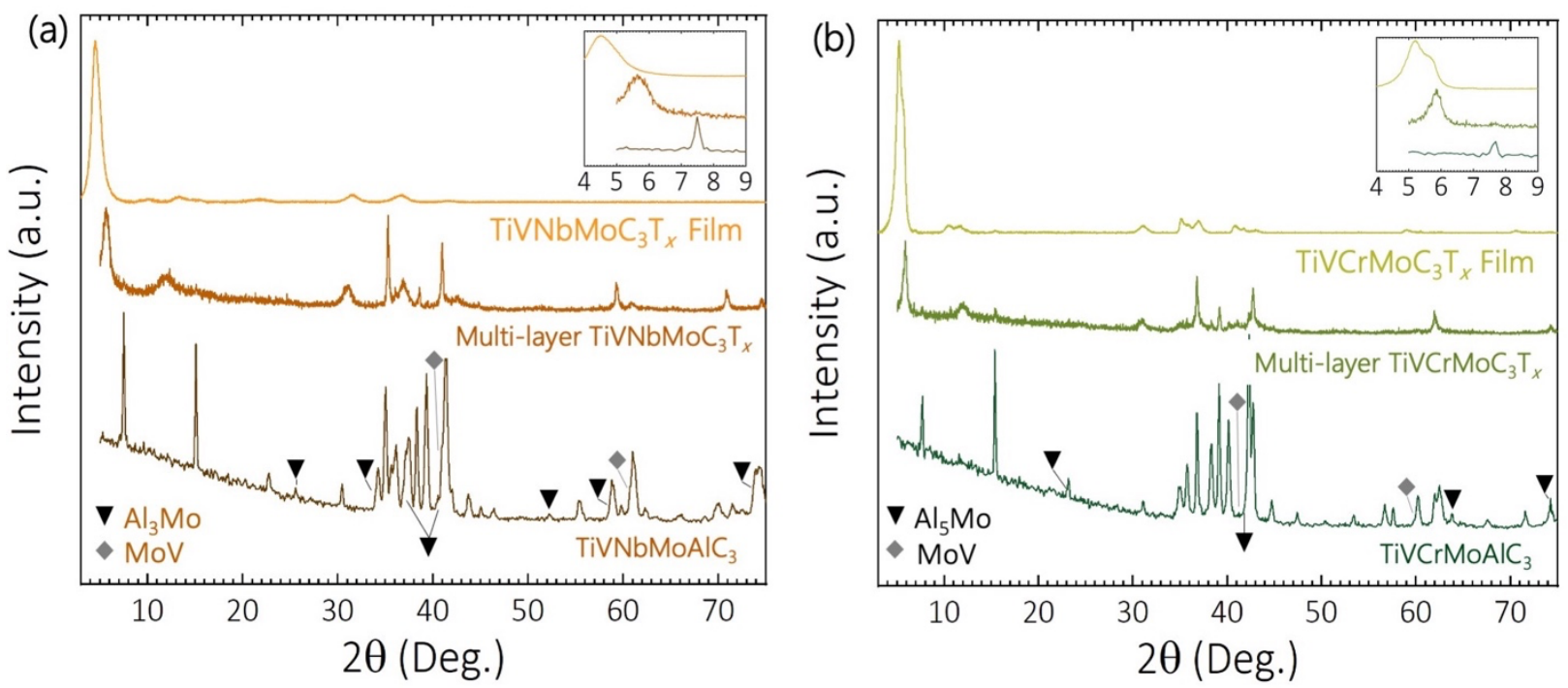

Figure 2. XRD patterns of high-entropy MAX phases and MXenes. (a) XRD patterns of $\mathrm{TiVNbMoAlC}_{3} \mathrm{MAX}$ and the resulting TiVNbMoC $3 T_{x}$ MXenes. (b) XRD patterns of TiVCrMoAlC 3 MAX and $\operatorname{TiVCrMoC}_{3} \mathrm{~T}_{x}$ MXenes. These plots illustrate the changes in diffraction pattern from their MAX phase precursor (bottom patterns) to their exfoliated multi-layer MXenes (middle patterns) followed by delamination into their single-to-few flake MXene films (top 
patterns). Inset on each panel illustrates the shifting of the (002) peak during the evolution from MAX to single-tofew layer MXene.

It is important to emphasize the effect of entropy stabilization and the use of four transition metals such as Ti-V-Nb-Mo or Ti-V-Cr-Mo combinations to synthesize stable, homogeneous, and single-phase $\mathrm{M}_{4} \mathrm{AC}_{3} \mathrm{MAX}$ structures. For example, not all combinations of these elements are found to be stable in double-transition metal (DTM) MAX phases. DTM MAX phases of $\mathrm{Cr}$ and Mo, such as $(\mathrm{Cr}, \mathrm{Mo})_{4} \mathrm{AlC}_{3}$, are predicted to be unstable both in random or ordered solid solution forms ${ }^{47}$ Additionally, for a Nb-Ti based $\mathrm{M}_{4} \mathrm{AlC}_{3}$ MAX phase, only $\left(\mathrm{Nb}_{0.8} \mathrm{Ti}_{0.2}\right)_{4} \mathrm{AlC}_{3}$ is reported and no equimolar $\mathrm{Nb}$-Ti-based $\mathrm{M}_{4} \mathrm{AlC}_{3}$ has been synthesized. The lack of binary transition metal MAX phases for all the constituent metals suggests that these phases are stabilized because of configurational entropy.

To further investigate the entropy stabilization, we studied the effect of less than the equimolar ratio of the four transition metals in $\mathrm{TiVNbMoAlC}_{3}$. To do so, three none-equimolar mixtures of the transition metals were prepared by lowering the molybdenum content from one to $0.7,0.4$, and 0.1. We mixed three ratios of Ti:V:Nb:Mo 1.1:1.1:1.1:0.7, 1.2:1.2:1.2:0.4, and 1.3:1.3:1.3:0.1 with Al:C 1.1:2.7 and sintered them at identical conditions to those for the equimolar ratio TiVNbMoAlC 3 MAX phase. However, the resulting composition was a mix of $\mathrm{M}_{4} \mathrm{AlC}_{3}$ and $\mathrm{M}_{2} \mathrm{AlC}$ MAX phases (Figure S1). While we have not characterized the composition of the undesired multiphases in the non-equimolar mixtures (Figure S1), they can possibly be a mixture of solid solution phases. For example, $\mathrm{M}_{2} \mathrm{AlC}$ may be comprised of a previously reported solid solution MAX phase of $\mathrm{Nb}$ and $\mathrm{V}^{17,50}$ and the $\mathrm{M}_{4} \mathrm{AlC}_{3}$ can contain either $\mathrm{Ti}^{-} \mathrm{Nb}^{51}$ or $\mathrm{Ti}^{5} \mathrm{~V}^{50}$ phases. The $\mathrm{M}_{4} \mathrm{AlC}_{3}$ phase might contain a non-equimolar ratio of all elements as well. Further analysis is needed to understand the stoichiometric ratio of the resulting undesired MAX phases. Regardless of the composition, simultaneous formation of two different MAX phases instead of a single-phase MPE TiVNbMoAlC 3 suggests that entropy controls the transition from multiphase to a highentropy single-phase, similar to other high-entropy alloys and ceramics. ${ }^{24,25}$ We will discuss the effect of entropy in the computational section of this paper. In general, the presence of a secondary competing MAX phase impurity, such as $\mathrm{M}_{2} \mathrm{AlC}$ in this case, creates a challenge in the successful synthesis of MXenes, because the topochemical etching process leads to a mix of two $\mathrm{M}_{2} \mathrm{CT}_{x}$ and $\mathrm{M}_{4} \mathrm{C}_{3} \mathrm{~T}_{\mathrm{x}}$ MXenes, instead of a phase pure $\mathrm{M}_{4} \mathrm{C}_{3} \mathrm{~T}_{\mathrm{x}}$ MXenes. 
To synthesize high-entropy MXenes, we used the aqueous hydrofluoric acid (HF) etching route, the most common synthesis method for MXenes. ${ }^{52}$ To do so, we added powders of $\mathrm{TiVNbMoAlC}_{3}$ and $\mathrm{TiVCrMoAlC}_{3}$ in $48 \% \mathrm{HF}$ for four days at $55^{\circ} \mathrm{C}$ to selectively etch $\mathrm{Al}$ atomic layers from the high-entropy MAX phases (Figure 1c) followed by repeated washing to a neutral $\mathrm{pH}$ (see Methods Section for more details). XRD patterns of the resulting dried powders (Figure 2a, b middle patterns) show a shift of the (002) peak in both phases to $5.65^{\circ}$ and $5.84^{\circ}$ for multilayer $\mathrm{TiVNbMoC}_{3} \mathrm{~T}_{x}$ and $\mathrm{TiVCrMoC}_{3} \mathrm{~T}_{x}$, respectively, which indicates an increase in the interflake spacing of 3.85 and $3.64 \AA$ respectively, compared to their MAX phase precursors. The increase in $c$-LPs and shift in the $(00 l)$ peak positions are in agreement with previous DTM MXene studies as an indication of exfoliation of $\mathrm{M}_{4} \mathrm{AlC}_{3}$ MAX phases to $\mathrm{M}_{4} \mathrm{C}_{3} \mathrm{~T}_{x}$ MXenes. ${ }^{48,51,53}$ The SEM images of the as-etched powders of $\mathrm{TiVNbMoC}_{3} \mathrm{~T}_{x}$ and $\mathrm{TiVCrMoC}_{3} \mathrm{~T}_{x}$ (Figure 3c, $\mathbf{d}$ ) show the accordion-like morphologies of multi-layer powders, which are usually observed for etched powders with high-concentration HF etching. ${ }^{3}$

To further delaminate the exfoliated high-entropy MXenes into single flakes of 2D MXenes, we used tetramethylammonium hydroxide $(\mathrm{TMAOH})$, which has been implemented for delamination of different MXene compositions. ${ }^{18,52,54}$ The delamination of $\mathrm{TiVNbMoC}_{3} \mathrm{~T}_{x}$ and $\mathrm{TiVCrMoC}_{3} \mathrm{~T}_{x}$ multilayer powders with TMAOH (details in Methods Section) resulted in black colloidal solutions (Figure S2). These solutions contain single-flake high-entropy MXenes (Figure 3e), which provides evidence of successful delamination of MXenes. By vacuum-assisted filtration of the resulting solutions, we fabricated high-entropy MXene films (Figure $\mathbf{3 f}$ and Figure S3). XRD patterns of the filtered films of $\mathrm{TiVNbMoC}_{3} \mathrm{~T}_{x}$ and $\mathrm{TiVCrMoC}_{3} \mathrm{~T}_{x}$ (Figure 2a and $\mathbf{b}$ top patterns) show only the basal plane peaks ( $00 l$ peaks), which indicates MXene flake restacking during filtration. The most intense (002) peak positions are at $4.51^{\circ}$ and $5.22^{\circ}$ for $\mathrm{TiVNbMoC}_{3} \mathrm{~T}_{x}$ and $\mathrm{TiVCrMoC}_{3} \mathrm{~T}_{x}$ films, respectively, which show an increased inter-flake distance of $7.81 \AA$ and $5.44 \AA$ as compared to the original MAX $c$-LPs spacing. This increase in inter-flake distance is in agreement with previous studies analyzing the shifting of the (002) peaks of MXene in single-to-few flake form. ${ }^{55}$ In addition, the (002) peak of $\mathrm{TiVCrMoC}_{3} \mathrm{~T}_{x}$ illustrates "double" (002) peaks, where the most intense peak is visualized at $5.22^{\circ}$ with a less-intense shoulder peak at $5.65^{\circ}$. These double peaks indicate the partial drying of water molecules from the MXene film. ${ }^{55}$ However, TiVCrMoC $3 \mathrm{~T}_{x}$ has low-intensity peaks between $31^{\circ}$ to $40^{\circ} 2 \theta$ which do not correspond to $(00 l)$ peaks. These peaks indicate non-basal plane reflections of MXene, which 
can be due to the remaining multi-layer flakes of MXene particles as seen using cross-sectional SEM methods on the $\mathrm{TiVCrMoC}_{3} \mathrm{~T}_{x} \mathrm{MXene}$ film (Figure 3f). However, the absence of non-basal plane reflections in the $\mathrm{TiVNbMoC}_{3} \mathrm{~T}_{x}$ film and the relative low intensity of non-basal plane peaks in comparison to the highly intense (002) peaks in $\mathrm{TiVCrMoC}_{3} \mathrm{~T}_{x}$ indicate that both films are either entirely or mostly, respectively, comprised of highly ordered single-to-few layers of high-entropy MXene flakes.

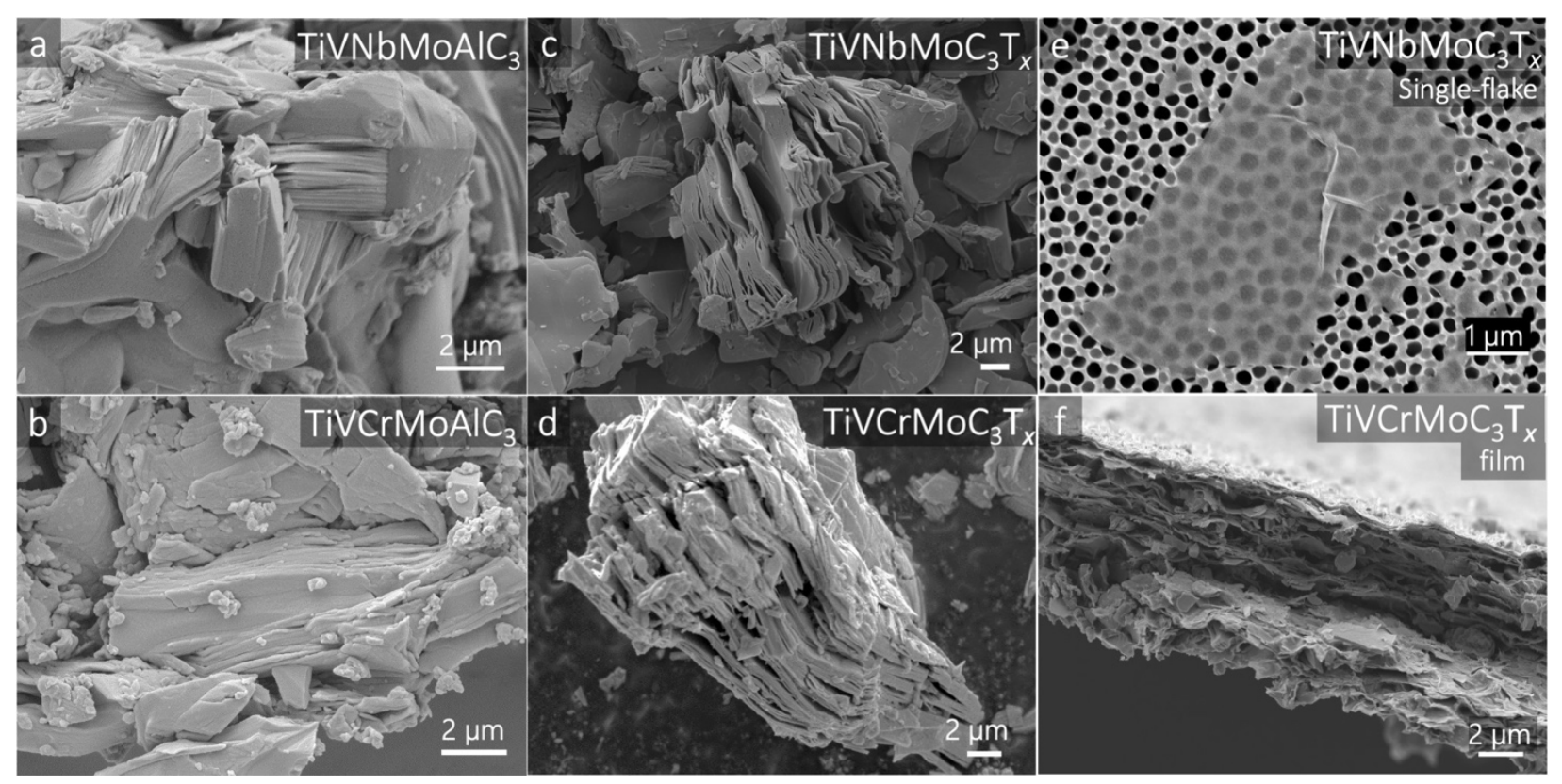

Figure 3. SEM micrographs of high-entropy MAX and MXenes. (a) TiVNbMoAlC 3 and (b) $\mathrm{TiVCrMoAlC}_{3}$ showing layered formation of the carbide structures typical of a MAX phase. (c, d) Etched multi-layer MXene powders of (c) $\mathrm{TiVNbMoC}_{3} \mathrm{~T}_{x}(\mathrm{~d}) \mathrm{TiVCrMoC}_{3} \mathrm{~T}_{x}$ with accordion like morphologies formed due to selective etching of A layered from MAX in high-concentrated HF, (e) Single film of $\mathrm{TiVNbMoC}_{3} \mathrm{~T}_{x}$ MXene flake on an alumina substrate, (f) a vacuumassisted filtered film of $\mathrm{TiVCrMoC}_{3} \mathrm{~T}_{x}$ MXene.

We next analyzed the composition of the synthesized high-entropy MXenes with EDS combined with SEM on multi-layer particles of high-entropy MXenes (Figure 3c, d). The EDS point scans on the multi-layer particles of $\mathrm{TiVNbMoC}_{3} \mathrm{~T}_{x}$ and $\mathrm{TiVCrMoC}_{3} \mathrm{~T}_{x}$ are presented in Table S1, which indicate that the MXene multi-layer sheets retain their transition metals stoichiometric ratios of the MAX phase precursors (Figure 3a, b) after HF etching. Our EDS results also show that Mo and Cr molar ratios in the measured multi-layer particles are slightly lower than those in their respective MAX precursors (Table S1). The slightly lower concentration of $\mathrm{Cr}$ in the multi-layer high-entropy MXenes may be attributed to relatively weaker $\mathrm{Cr}-\mathrm{C}$ bonds 
compare to other $\mathrm{M}-\mathrm{C}$ bonds ${ }^{56}$ and the more reactive nature of $\mathrm{Cr}$-containing $\mathrm{MXenes}$ during selective etching. ${ }^{18}$ The etching conditions used here, $50 \% \mathrm{HF}$ for $96 \mathrm{~h}$ at $55{ }^{\circ} \mathrm{C}$, are among the harshest conditions used for the synthesis of MXenes. ${ }^{57}$ Additionally, while our results indicate favorable entropy stabilization, we cannot rule out the tendency of Mo and $\mathrm{Cr}$ to prefer the outer transition metal atomic layers in a MXene 2D flake, as was observed in ordered double transition metal MAX and MXene phases. ${ }^{12,58-63}$ The possible preferred atomic positions of Mo and Cr can expose them directly to HF during etching, which leads to their relatively higher removal rates while creating their transition metal vacancies in the outer M layers. ${ }^{64}$ Future studies are needed to probe any possibility of ordering in these MPE MXenes.

We further analyzed the compositions and chemical bonding within our high-entropy MXenes by conducting X-ray photoelectron spectroscopy (XPS) analysis to evaluate the chemical states as well as the coordination of the transition metals and carbon within the high-entropy $\mathrm{TiVNbMoC}_{3} \mathrm{~T}_{x}$ and $\mathrm{TiVCrMoC}_{3} \mathrm{~T}_{x}$ MXenes. To gain an average distribution of $\mathrm{Ti}, \mathrm{V}, \mathrm{Nb} / \mathrm{Cr}, \mathrm{Mo}$, and $\mathrm{C}$, we set the spot size to $400 \mu \mathrm{m}$ for all XPS analyses. Figure 4a-f shows the XPS high-resolution spectra for the top surface of as-prepared $\mathrm{TiVNbMoC}_{3} \mathrm{~T}_{x}$ and $\mathrm{TiVCrMoC}_{3} \mathrm{~T}_{x} \mathrm{MXenes}$. The $\mathrm{Cl}$ s highresolution spectra highlight the existence of $\mathrm{C}-\mathrm{Mo} / \mathrm{Ti}-\mathrm{T}_{x}, \mathrm{C}-\mathrm{Ti} / \mathrm{MoT}_{x}, \mathrm{C}-\mathrm{C}-\mathrm{CH}_{x}, \mathrm{C}-\mathrm{O}$, and $\mathrm{COO}$ moieties in both structures. The complex nature of this chemical bonding was also seen in other MXenes, including titanium carbide (e.g., $\left.\mathrm{Ti}_{3} \mathrm{C}_{2} \mathrm{~T}_{x}\right)$ and niobium carbide $\left(\mathrm{Nb}_{2} \mathrm{CT}_{x}\right){ }^{65-67}$ The Ti2p regions for both $\mathrm{TiVNbMoC}_{3} \mathrm{~T}_{x}$ and $\mathrm{TiVCrMoC}_{3} \mathrm{~T}_{x}$ highlights the coordination of $\mathrm{Ti}$ as Ti-C and $\mathrm{TiO}_{2}$ (note that $\mathrm{TiO}_{2}$ forms due to surface oxidation of the MXene). The V2p region suggests that $\mathrm{V}$ is coordinated via $\mathrm{V}^{2+} / \mathrm{V}^{4+}$ moieties or as $\mathrm{V}_{2} \mathrm{O}_{3}$, while the Mo3d region indicates that Mo exists as $\mathrm{Mo}^{5+} / \mathrm{Mo}^{6+}, \mathrm{C}-\mathrm{Mo}-\mathrm{T}_{x}$, and Mo in both structures. For $\mathrm{TiVNbMoC}_{3} \mathrm{~T}_{x}$, the $\mathrm{Nb}$ coordination is composed of several species, including $\mathrm{Nb}^{0}, \mathrm{Nb}$ (I, II, or IV) $\mathrm{NbO}, \mathrm{Nb}^{3+}-\mathrm{O}, \mathrm{Nb}^{4+}-\mathrm{O}$, and $\mathrm{Nb}_{2} \mathrm{O}_{5}$, and while in $\mathrm{TiVCrMoC}_{3} \mathrm{~T}_{x}$, the $\mathrm{Cr}$ coordinates as $\mathrm{Cr}^{2+}$ and $\mathrm{Cr}^{0}$. Full details regarding the spectral deconvolutions can be found in Tables $\mathbf{S 2}$ and $\mathbf{S 3}$.

To further probe the distribution of $\mathrm{Ti}, \mathrm{V}, \mathrm{Nb} / \mathrm{Cr}, \mathrm{Mo}$, and $\mathrm{C}$ in $\mathrm{TiVNbMoC}_{3} \mathrm{~T}_{x}$ and $\mathrm{TiVCrMoC}_{3} \mathrm{~T}_{x}$, we completed XPS depth profiles with $\mathrm{Ar}^{+}$etching (Figure 4g, h). While the surface of the MXene films was slightly oxidized, the relative magnitude of the elemental deviation was more apparent after 20-30 s of $\mathrm{Ar}^{+}$etching, which agrees with our EDS results on the multilayer MXenes (Table S1). Several important conclusions can be drawn from the XPS depth 
profiling. Quantitatively, the distribution of $\mathrm{Ti}, \mathrm{V}, \mathrm{Nb} / \mathrm{Cr}$, Mo, and $\mathrm{C}$ was relatively constant through the MXene films, suggesting that the MXenes have a relatively uniform distribution of the transition metals in the structure. With increasing depth, the concentration of each element approaches the theoretical concentration of the high-entropy MXenes as observed in their precursor MAX phases.
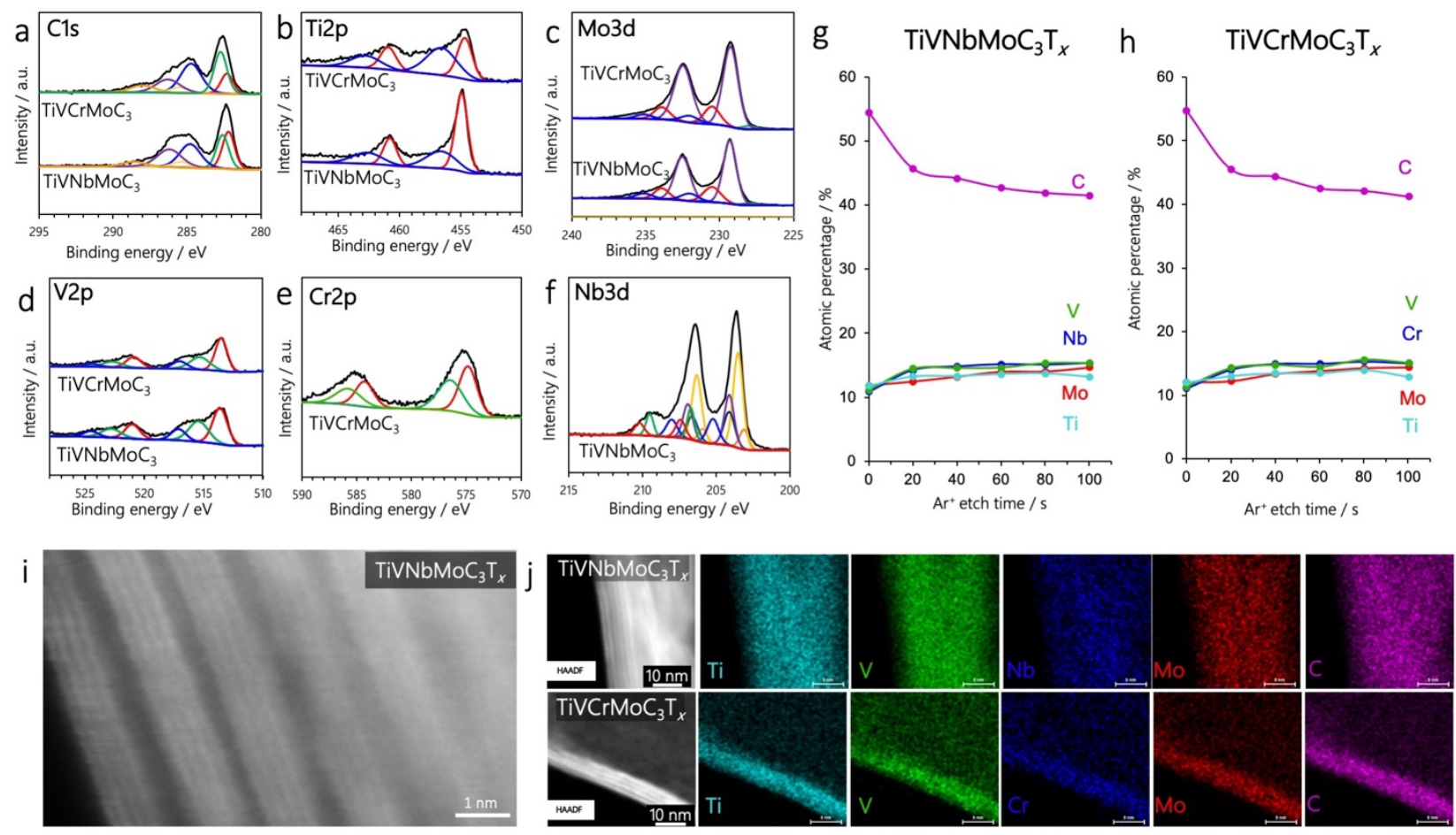

Figure 4. XPS, and STEM with EDS results of high-entropy MXenes. (a-f) XPS spectra of $\mathrm{TiVNbMoC}_{3} \mathrm{~T}_{x}$ and $\mathrm{TiVCrMoC}_{3} \mathrm{~T}_{x} .(\mathrm{g}, \mathrm{h})$ Atomic \% vs $\mathrm{Ar}^{+}$etching time for both MXenes. Equi-proportional At $\mathrm{wt} \%$ of the principal elements with increase etch rates indicate uniform distribution of the atoms in the inner layer of MXenes. (i) Highangle annular dark-field (HAADF) STEM images of TiVNbMoC $3 \mathrm{~T}_{x}$ MXene. (j) HAADF STEM combined with EDS results of $\mathrm{TiVNbMoC}_{3} \mathrm{~T}_{x}$ (top) and $\mathrm{TiVCrMoC}_{3} \mathrm{~T}_{x}$ (bottom) indicating uniform layered morphology and atomic distribution of $\mathrm{Ti}, \mathrm{V}, \mathrm{Nb} / \mathrm{Cr}, \mathrm{Mo}, \mathrm{C}$ atoms.

To further characterize the composition of the synthesized high-entropy MXenes, scanning transmission electron microscopy (STEM) analysis combined with EDS was performed to better understand the elemental distribution and nanostructure of $\mathrm{TiVNbMoC}_{3} \mathrm{~T}_{x}$ and $\mathrm{TiVCrMoC}_{3} \mathrm{~T}_{x}$ flakes to confirm their 4-layer structure and map their elements. To accurately probe the structure of these MXenes, both samples were drop-cast on a lacey carbon-coated $\mathrm{Cu}$ grid (see Methods Section for more details). Figure 4i shows high-angle annular dark-field (HAADF) STEM image of $\mathrm{TiVNbMoC}_{3} \mathrm{~T}_{x}$, indicating that each MXene flake is composed of four transition metal atomic 
layers, in agreement with previously reported STEM of $\mathrm{M}_{4} \mathrm{C}_{3} \mathrm{~T}_{x}$ MXenes. ${ }^{12,}{ }^{48}$ EDS mapping (Figure 4j and S4) of each MXene compositions reveals that $\mathrm{Ti}, \mathrm{V}, \mathrm{Nb} / \mathrm{Cr}, \mathrm{Mo}$, and $\mathrm{C}$ are distributed across the transition metal layers, signifying the near equimolar distribution of the transition metals in high-entropy MXenes.

To understand the synthesizability of high-entropy MAX and MXene phases as well as to quantify the thermodynamic stability of the MAX compositions, we investigated the formation enthalpy $(\Delta H)$ of the MAX compositions with reference to the combination of most competitive

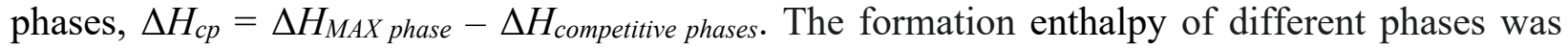
calculated using the total energy of those phase structures and the total energies (per atom) of the $\mathrm{M}, \mathrm{A}$, and $\mathrm{X}$ elements in their standard state (bulk phases). The most competing phases were then identified using a linear optimization process. ${ }^{68}$ The negative $\Delta H_{c p}$ specifies the relative stability of MAX phases with respect to the competitive phases. In general, the more negative enthalpy values indicate higher possibility for their experimental realization. The compositions of the MAX phases along with their enthalpies of formation as well as lattice parameters are given in TableS4. The calculated electron concentration of the already synthesized MAX phases with reference to their relative formation energies is shown in Figure S5.

In multicomponent systems, a major contributor to their stabilization arises from configurational entropy i.e. statistically the number of discrete representative positions of the alloy constituents. The entropic contribution for the ternary and quaternary MAX systems can be calculated using: ${ }^{69}$

$$
\Delta S_{m i x}=-R \sum_{i=1}^{k} x_{i} \ln x_{i}
$$

Where $x_{i}$ is the mole fraction of the $i^{\text {th }}$ component in a system with $\mathrm{k}$ total components. ${ }^{22}$ The calculated entropic contribution at $1600{ }^{\circ} \mathrm{C}$ for ternary and quaternary systems are $-0.1773 \mathrm{eV} /$ f.u. and $-0.2238 \mathrm{eV} /$ f.u. respectively. Based on configurational entropy, the four-element MAX phase has more favorable entropy to form as a single-phase compared to the three-element one (Figure S6). The entropic stabilization explains the preference for four-element single-phase MAX phase as compared to three-element phases of MAX under similar synthesis conditions. Note, however, that our enthalpy calculations reveal that the three-element MAX is also synthesizable (Table S4). Since we only used one synthesis temperature and duration $\left(1600{ }^{\circ} \mathrm{C}-4 \mathrm{~h}\right)$ for the sake of consistency, we cannot rule out the single-phase formation of the three-element MAX by further 
annealing at a desired temperature and duration. It is known that a lower contribution of configurational entropy can lead to the formation of a multiphase system (undesired phases) in the absence of post-annealing treatment. ${ }^{25}$ Further detailed studies on the nature of competing phases and formation pathways are required to understand the trends in synthesizability of high-entropy MAX phases.

\section{CONCLUSIONS AND FUTURE OUTLOOK}

In summary, we have synthesized the first high-entropy $\mathrm{TiVNbMoAlC}_{3}$ and $\mathrm{TiVCrMoAlC}_{3}$ MAX phases and turn them to high-entropy TiVNbMoC $3 \mathrm{~T}_{x}$ and $\mathrm{TiVCrMoC}_{3} \mathrm{~T}_{x}$ MXenes with an equimolar proportion of Ti:V:Nb:Mo and Ti:V:Cr:Mo principal transition metals. We formed these high-entropy MAX phases using traditional pressureless reactive sintering and synthesized their respective single-to-few layer MXenes utilizing hydrofluoric acid-based selective etching followed by tetramethylammonium hydroxide delamination. We confirmed the synthesis and purity of our high-entropy MAX phases and their successful synthesis to single-to-few layer highentropy MXenes using XRD, SEM and STEM. In addition, we confirmed the bonding characteristics in high-entropy MXenes using XPS and identified the equimolar composition of transition metals utilizing EDS in SEM. We further establish the equimolar distribution of transition metals in the transition metal layers in these MXenes using elemental mapping with EDS in STEM. Our first-principles calculations trace the synthesizability of quaternary high-entropy MAX to an entropy driven stabilization and highlight the importance of high configurational entropy in equimolar multi-elements in forming pure and stable phases of MPE MAX phases. We thus report on the successful synthesis of high-entropy MAX and MXene materials by maximizing the configurational entropy to stabilize (near) equimolar mixtures in a fashion analogous to that in other disordered multicomponent systems (e.g. ceramics and metals). In general, synthesis of this new subgroup of high-entropy MPE MXenes adds a large possible compositional space to add to the growing family of MXenes.

We have laid the groundwork for further studies to focus on a comprehensive analysis of more compositions of high-entropy MAX phases and their derived MXenes to further analyze stable compositions using both computational and experimental methods. Furthermore, an understanding of the control of the composition of each transition metal layers of MXenes could also play a significant role in future tailoring of their electrochemical, catalytic, electrical, magnetic 
properties. election of multiple constituent transition metals can further enable the development of topological insulator MXenes, which have not been synthesized yet but are predicted in theoretical studies, ${ }^{70,71}$ specifically due to larger degrees of freedom in component selectivity and phase formation using more than one transition metals in MXenes.

From a computational perspective, the enormous compositional space coupled with the possibility of entropic stabilization in high-entropy MAX and MXene phases opens several new avenues as well as challenges. Traditional modeling approaches can be combined with recent advances in big data analytics to answer several new intriguing questions related to quantitative calculation of the entropy, prediction of thermochemical properties, the transition temperatures, and investigation of their transport properties to better understand the mechanisms affecting their synthesizability. We envision many opportunities for Monte Carlo simulations in computing entropic contributions - this would require many energetic contributions that would benefit from cluster expansion and other machine learning methods that build surrogate models based on abinitio training data. The kinetic aspects could be probed with molecular dynamics that can shed light on the diffusion rates in disordered materials. Newer data driven approaches can also provide useful information on the lifetimes and stability of metastable phases under different operating conditions. Overall, the vast quantity of synthetic and experimental datasets that will be generated creates tremendous opportunities for artificial intelligence and machine learning to identify critical trends for accelerating materials design and materials discovery in this important class of highentropy 2D materials.

\section{METHODS SECTION}

Synthesis of high-entropy MAX phases. All elements Ti (325 mesh), V (325 mesh), Mo (250 mesh), $\mathrm{Nb}$ (325 mesh), Cr (325 mesh), Al(325 mesh), C (calcinated coke) (325 mesh), and reagents HF (48 wt\% aqueous), Tetramethylammonium hydroxide (TMAOH) $(25 \% \mathrm{w} / \mathrm{w}$ aqueous) were obtained from Fisher Scientific and used without further processing unless specified.

For $\mathrm{Al}$ and $\mathrm{C}$, we followed the typical ratios that we always use for the synthesis of $\mathrm{M}_{4} \mathrm{AlC}_{3}{ }^{48,58}$ In general, we add additional amount $(\sim 0.1$ moles) of Al to compensate for any evaporation during the MAX synthesis. ${ }^{49,72}$ We also use less than stoichiometric ratio of $\mathrm{C}$, as we know all MAX phases are carbon deficient similar to binary transition metal carbides. ${ }^{49,73}$ 
Molar ratios of the elemental powders for $\mathrm{Ti} / \mathrm{V} / \mathrm{Nb} / \mathrm{Mo} / \mathrm{Al} / \mathrm{C}$ and $\mathrm{Ti} / \mathrm{V} / \mathrm{Cr} / \mathrm{Mo} / \mathrm{Al} / \mathrm{C}$ were 1:1:1:1:1.1:2.7 respectively, and the blends were milled in polycarbonate jars on a tumbler mill with zirconia balls at a powder to ball weight ratios of 1:5 for $18 \mathrm{~h}$ at $60 \mathrm{rpm}$. Ball milled powders were transferred to alumina crucibles and sintered in a conventional tube furnace (Carbolite Gero) fitted with an alumina tube at 1600 ${ }^{\circ} \mathrm{C}$, held for $4 \mathrm{~h}$. The temperature ramp rate was $3.5^{\circ} \mathrm{C} / \mathrm{min}$. The furnace was flushed with Ar gas for 10-15 min prior to firing of the MAX powders. A constant Ar flow was maintained throughout the run till the samples reached room temperature. Post cooling, the synthesized MAX phases were drilled with a TiNcoated drill bit and sieved with a $40 \mu \mathrm{m}$ sieve to obtained uniform grains of MAX powder for etching.

Synthesis and delamination of high-entropy MXenes. MXenes (both $\mathrm{TiVNbMoC}_{3}$ and $\mathrm{TiVCrMoC}_{3}$ ) were synthesized via top-down synthesis, by selective etching of their respective MAX phases. In a typical experiment, $2 \mathrm{~g}$ of MAX was added slowly ( $\sim 60 \mathrm{~s}$ ) to a polycarbonate jar filled with $20 \mathrm{ml}$ of HF (48 wt\%) and held at $55^{\circ} \mathrm{C}$ with continuous stirring at 400 RPM for 4 days. The etched MXene was washed repeatedly 4-5 times ( $250 \mathrm{ml}$ with DI water) in a centrifuge at $4200 \mathrm{RPM}$ with each typical run lasting 3-5 min. The $\mathrm{pH}(>6)$ neutralized etched MXene cakes were obtained via vacuum-assisted filtration with a 2.5 micron cellulose filter paper. The etching process is identical for both $\mathrm{TiVNbMoAlC}_{3}$ and $\mathrm{TiVCrMoAlC}_{3}$ phases. Filtered MXene cakes were subsequently delaminated with $5 \mathrm{wt} \%$ TMAOH with continuous stirring at 500$600 \mathrm{RPM}$ for $4 \mathrm{~h}$ at $55^{\circ} \mathrm{C}$. The delaminated MXenes were washed repeatedly for 5 times $(\sim 250 \mathrm{ml}$ with DI water) in a centrifuge at $10000 \mathrm{RPM}$ with variable times $(5-10 \mathrm{~min})$ to bring the $\mathrm{pH}$ to $\sim 6$. The supernatant was collected and films were made by vacuum-assisted filtration. Figure S4 shows the supernatant, clay, and films of $\mathrm{TiVCrMoC}_{3}$ and $\mathrm{TiVNbMoC}_{3}$ MXenes.

\section{Microstructural Characterization.}

XRD. The structural characteristics of the MAX phases and MXenes were characterized using a Bruker D8 Discover x-ray diffractometer with a $\mathrm{Cu} \mathrm{K}$ (alpha) radiation wavelength of $1.54184 \AA$ paired with the Vantec two-dimensional detector $\left(\mathrm{XRD}^{2}\right)$. The scans were carried out from $5-75 \operatorname{deg} 2 \theta\left(0-90^{\circ}\right)$ using step sizes of $5^{\circ} 2 \theta$ with a time step of $60 \mathrm{~s} / \mathrm{step}$. MAX phase and multi-layer MXene powders were placed into cylindrical holes on an aluminum substrate with dimensions $5 \mathrm{~mm}$ diameter $\mathrm{x} 1.5 \mathrm{~mm}$ depth. The MAX powders were preferentially ordered using a clean glass slide for pressing on the top of the powder samples. $\mathrm{TiVNbMoC}_{3}$ and $\mathrm{TiVCrMoC}_{3} \mathrm{MXene}$ single-to-few layer films were cut into $5 \mathrm{~mm}$ x $10 \mathrm{~mm}$ rectangular pieces and one of these film pieces was stacked on an amorphous double-sided carbon tape on a clean glass substrate. The sample height was aligned using dual-focused laser beams. Spectral data was analyzed with crystal impact software, Match!

SEM/EDS. A JEOL JSM-7800F FESEM equipped with an in-lens thermal field emission electron gun and a conical objective lens with in-tandem upper and lower electron detectors was used to collect morphological and compositional data of the samples. All specimens were sputter-coated with gold (Denton 
Desk V Turbo) to enhance conduction on the surface. Energy Dispersion Spectroscopy was performed via an EDAX Octane Super Detector and associated EDAX TEAM software. The working distance (10 mm) and elevation angle $\left(35^{\circ}\right)$ were fixed along with a scale setting of 58 for the EDS analyses. the dead time for each run was manually adjusted to 27-35. A random spot was selected and was analyzed point-wise (at 10 spots) with an excitation voltage of $15-25 \mathrm{kV}$ and a peak current of 8-10 Amp. A Magnification of $1000 \mathrm{kX}$ was used for obtaining the composition of the MXene films.

XPS and STEM/EDS. X-ray photoelectron spectroscopy (XPS) spectra were collected for each powder sample on a Thermo K-alpha XPS system with a spot size of $400 \mu \mathrm{m}$ at an energy resolution of $0.1 \mathrm{eV}$. Ar ${ }^{+}$ sputtering was carried out with a beam energy of $4 \mathrm{eV}$ and the cluster size was 1000 atoms. For the XPS depth profiles, the atomic percentages were calculated for only $\mathrm{Ti}, \mathrm{V}, \mathrm{Mo}, \mathrm{Cr} / \mathrm{Nb}$, and $\mathrm{C}$. All XPS spectra were analyzed using Thermo Avantage, a software package provided through ThermoScientific. Scanning transmission electron microscopy (STEM) was performed on an FEI Talos TEM/STEM equipped with an EDS detector (Bruker) operated at $200 \mathrm{kV}$. Elemental maps were collected with a STEM spot size of 6. All $\mathrm{S} / \mathrm{TEM}$ specimen were prepared by dispersing MXene-based materials in D.I. $\mathrm{H}_{2} \mathrm{O}$ and drop-casting the dispersion onto lacey carbon-coated copper grids.

First-principles calculations. To simulate the chemical disorder of actual high entropy MAX while maintaining a tractable size for the computational cell, we used special quasi-random structures (SQSs) as implemented in the ATAT software package. ${ }^{74}$ For the quaternary MAX phase, we utilized a $2 \times 2 \times 1$ supercell whereas in case of ternary system, a supercell of $3 \times 3 \times 1$ from the parent MAX phase was used. The Perdue-Burke-Ernzerhof $(\mathrm{PBE})^{75}$ exchange-correlation functional and the projected augmented wave approach ${ }^{45,46}$ were used through its implementation in the Vienna Ab-Initio Simulation Package (VASP). ${ }^{76-}$ ${ }^{78}$ The MAX phase structures were fully optimized through relaxation of the unit-cell shape, atomic positions, and volume using the conjugate gradient method until the maximum residual force acting on each atom became less than $0.01 \mathrm{eV} / \AA$. The electronic energy convergence criterion used is $10^{-6} \mathrm{eV} /$ cell having a plane wave cut-off energy of $520 \mathrm{eV}$. The Brillouin zone was integrated using Monkhorst-Pack k-point sampling method. ${ }^{79}$ A dense k-point grid was employed, defined by $\mathrm{n}_{\text {atoms }} \times \mathrm{n}_{k \text {-points }} \approx 1000$, where $\mathrm{n}_{\text {atoms }}$ is the number of atoms in the primitive cell and $\mathrm{n}_{k \text {-points }}$ being the number of $\mathrm{k}$-points respectively. ${ }^{80}$ 


\section{Supporting Information:}

XRD of Ti:V:Nb:Mo:Al:C 1.33:1.33:1.33:0.1:1.1:2.7, 1.2:1.2:1.2:0.4:1.1:2.7 and 1.1:1.1:1.1:0.7:1.1:2.7, digital photos of different stages of $\mathrm{TiVCrMoC}_{3} \mathrm{~T}_{x}$ and $\mathrm{TiVNbMoC}_{3} \mathrm{~T}_{x}$ synthesis, $\mathrm{SEM}$ images of $\mathrm{TiVNbMoC}_{3} \mathrm{~T}_{x}$ and $\mathrm{TiVCrMoC}_{3} \mathrm{~T}_{x}$ MXene films, HAADF STEM images of $\mathrm{TiVNbMoC}_{3} \mathrm{~T}_{x}$ and $\mathrm{TiVCrMoC}_{3} \mathrm{~T}_{x}$ flakes, and EDS map of $\mathrm{TiVCrMoC}_{3} \mathrm{~T}_{x}$ flakes, tables of EDS analysis of high-entropy MXene films in SEM, XPS deconvolution data with Binding Energy $\mathrm{TiVNbMoC}_{3} \mathrm{~T}_{x}$ and $\mathrm{TiVCrMoC}_{3} \mathrm{~T}_{x}$, calculated enthalpy of formation of TiVCrMoAlC 3 , TiVNbMoAlC $3,\left(\mathrm{Ti}_{1.33} \mathrm{~V}_{1.33} \mathrm{Nb}_{1.33}\right) \mathrm{AlC}_{3} \mathrm{MAX}$ phases, and calculated electron concentration of the already synthesized MAX phases with reference to their relative formation energy, configurational entropy of binary, ternary, and quaternary phases as a function of the last constituent $\mathrm{mol} \%$.

\section{Notes}

The authors declare no competing financial interest.

Acknowledgments: B.A. acknowledge startup funding from the Department of Mechanical and Energy Engineering and Purdue School of Engineering and Technology at IUPUI. Z.D.H. was supported by Laboratory Directed Research and Development (LDRD) funding from Argonne National Laboratory, provided by the Director, Office of Science, of the U.S. Department of Energy under Contract No. DE-AC02-06CH11357. This computational material is based upon work supported by the U.S. Department of Energy, Office of Science, Office of Basic Energy Sciences Data, Artificial Intelligence and Machine Learning at DOE Scientific User Facilities program under Award Number 34532. Use of the Center for Nanoscale Materials, an Office of Science user facility, was supported by the U.S. Department of Energy, Office of Science, Office of Basic Energy Sciences, under Contract No. DE-AC02-06CH11357. This research used resources of the National Energy Research Scientific Computing Center, which was supported by the Office of Science of the U.S. Department of Energy under Contract No. DE-AC0205CH11231. An award of computer time was provided by the Innovative and Novel Computational Impact on Theory and Experiment (INCITE) program of the Argonne Leadership Computing Facility at the Argonne National Laboratory, which was supported by the Office of Science of the U.S. Department of Energy under Contract No. DE-AC02-06CH11357. SKRS acknowledges UIC start-up funds for supporting this research. BZ acknowledges the support from Henan Key Laboratory of Photovoltaic Materials, Henan University, Kaifeng, China. We would also like to acknowledge the use of JEOL7800f Field Emission Scanning Electron Microscope, which was awarded through NSF grant MRI-1229514, and for use of Bruker XRD equipment, which was awarded through NSF grant MRI-1429241. 


\section{References}

1. Naguib, M.; Mashtalir, O.; Carle, J.; Presser, V.; Lu, J.; Hultman, L.; Gogotsi, Y.; Barsoum, M. W., Two-Dimensional Transition Metal Carbides. ACS Nano 2012, 6 (2), 1322-1331.

2. $\quad$ Naguib, M.; Kurtoglu, M.; Presser, V.; Lu, J.; Niu, J.; Heon, M.; Hultman, L.; Gogotsi, Y.; Barsoum, M. W., Two-Dimensional Nanocrystals Produced by Exfoliation of $\mathrm{Ti}_{3} \mathrm{AlC}_{2}$. Advanced Materials 2011, 23 (37), 4248-4253.

3. Anasori, B.; Lukatskaya, M. R.; Gogotsi, Y., 2D metal carbides and nitrides (MXenes) for energy storage. Nature Reviews Materials 2017, 2 (2), 16098.

4. Deysher, G.; Shuck, C. E.; Hantanasirisakul, K.; Frey, N. C.; Foucher, A. C.; Maleski, K.; Sarycheva, A.; Shenoy, V. B.; Stach, E. A.; Anasori, B., Synthesis of Mor VAlC 4 MAX Phase and TwoDimensional $\mathrm{Mo}_{4} \mathrm{VC}_{4}$ MXene with 5 Atomic Layers of Transition Metals. ACS Nano 2019, 14, 204-217.

5. Wang, H.-W.; Naguib, M.; Page, K.; Wesolowski, D. J.; Gogotsi, Y., Resolving the Structure of $\mathrm{Ti}_{3} \mathrm{C}_{2} \mathrm{~T}_{\mathrm{x}}$ MXenes through Multi-Level Structural Modeling of the Atomic Pair Distribution Function. Chemistry of Materials 2015, 28 (1), 349-359.

6. $\quad$ Gogotsi, Y.; Anasori, B., The Rise of MXenes. ACS Nano 2019, 13 (8), 8491-8494.

7. Khaledialidusti, R.; Khazaei, M.; Khazaei, S.; Ohno, K., High-throughput computational discovery of ternary-layered MAX phases and prediction of their exfoliation for formation of 2D MXenes. Nanoscale 2021.

8. Mathis, T.; Maleski, K.; Goad, A.; Sarycheva, A.; Anayee, M.; Foucher, A. C.; Hantanasirisakul, K.; Stach, E.; Gogotsi, Y.; Alexandre, C., Modified MAX Phase Synthesis for Environmentally Stable and Highly Conductive $\mathrm{Ti}_{3} \mathrm{C}_{2}$ MXene. ChemRxiv 2020.

9. Lipatov, A.; Alhabeb, M.; Lu, H.; Zhao, S.; Loes, M. J.; Vorobeva, N. S.; Dall'Agnese, Y.; Gao, Y.; Gruverman, A.; Gogotsi, Y.; Sinitskii, A., Electrical and Elastic Properties of Individual SingleLayer $\mathrm{Nb}_{4} \mathrm{C}_{3} \mathrm{~T}_{x}$ MXene Flakes. Advanced Electronic Materials 2020, 6 (4), 1901382.

10. Lim, K. R. G.; Handoko, A. D.; Nemani, S. K.; Wyatt, B.; Jiang, H.-Y.; Tang, J.; Anasori, B.; Seh, Z. W., Rational Design of Two-Dimensional Transition Metal Carbide/Nitride (MXene) Hybrids and Nanocomposites for Catalytic Energy Storage and Conversion. ACS Nano 2020, 14 (9), 10834-10864.

11. Verger, L.; Xu, C.; Natu, V.; Cheng, H.-M.; Ren, W.; Barsoum, M. W., Overview of the synthesis of MXenes and other ultrathin 2D transition metal carbides and nitrides. Current Opinion in Solid State and Materials Science 2019, 23 (3), 149-163.

12. Anasori, B.; Xie, Y.; Beidaghi, M.; Lu, J.; Hosler, B. C.; Hultman, L.; Kent, P. R. C.; Gogotsi, Y.; Barsoum, M. W., Two-Dimensional, Ordered, Double Transition Metals Carbides (MXenes). ACS Nano 2015, 9 (10), 9507-9516.

13. Tao, Q.; Dahlqvist, M.; Lu, J.; Kota, S.; Meshkian, R.; Halim, J.; Palisaitis, J.; Hultman, L.; Barsoum, M. W.; Persson, P. O.; Rosen, J., Two-dimensional Mo ${ }_{1.33} \mathrm{C}$ MXene with divacancy ordering prepared from parent 3D laminate with in-plane chemical ordering. Nature Communications 2017, 8.

14. Maleski, K.; Shuck, C. E.; Fafarman, A. T.; Gogotsi, Y., The Broad Chromatic Range of TwoDimensional Transition Metal Carbides. Advanced Optical Materials 2021, 9 (4), 2001563.

15. Han, M.; Shuck, C. E.; Rakhmanov, R.; Parchment, D.; Anasori, B.; Koo, C. M.; Friedman, G.; Gogotsi, Y., Beyond $\mathrm{Ti}_{3} \mathrm{C}_{2} \mathrm{~T}_{x}$ : MXenes for Electromagnetic Interference Shielding. ACS Nano 2020, 14 (4), 5008-5016.

16. Hong, W.; Wyatt, B. C.; Nemani, S. K.; Anasori, B., Double transition-metal MXenes: Atomistic design of two-dimensional carbides and nitrides. MRS Bulletin 2020, 45 (10), 850-861.

17. Han, M.; Maleski, K.; Shuck, C. E.; Yang, Y.; Glazar, J. T.; Foucher, A. C.; Hantanasirisakul, K.; Sarycheva, A.; Frey, N. C.; May, S. J.; Shenoy, V. B.; Stach, E. A.; Gogotsi, Y., Tailoring Electronic and Optical Properties of MXenes through Forming Solid Solutions. Journal of the American Chemical Society 2020, 142 (45), 19110-19118.

18. Hantanasirisakul, K.; Anasori, B.; Nemsak, S.; Hart, J. L.; Wu, J.; Yang, Y.; Chopdekar, R. V.; Shafer, P.; May, A. F.; Moon, E. J.; Zhou, J.; Zhang, Q.; Taheri, M. L.; May, S. J.; Gogotsi, Y., Evidence of a magnetic transition in atomically thin $\mathrm{Cr}_{2} \mathrm{TiC}_{2} \mathrm{~T}_{x}$ MXene. Nanoscale Horizons 2020, 5 (12), 1557-1565. 
19. Khazaei, M.; Ranjbar, A.; Arai, M.; Yunoki, S., Topological insulators in ordered double transition metals $\mathrm{M}_{2}{ }_{2} \mathrm{M}^{\prime \prime} \mathrm{C}_{2}\left(\mathrm{M}^{\prime}=\mathrm{Mo}, \mathrm{W}\right.$; M"= Ti, Zr, Hf) MXenes. Physical Review B 2016, 94 (12), 125152.

20. Yeh, J. W.; Chen, S. K.; Lin, S. J.; Gan, J. Y.; Chin, T. S.; Shun, T. T.; Tsau, C. H.; Chang, S. Y., Nanostructured High-Entropy Alloys with Multiple Principal Elements: Novel Alloy Design Concepts and Outcomes. Advanced Engineering Materials 2004, 6 (5), 299-303.

21. Cantor, B.; Chang, I. T. H.; Knight, P.; Vincent, A. J. B., Microstructural development in equiatomic multicomponent alloys. Materials Science and Engineering: A 2004, 375-377, 213-218.

22. Ye, Y. F.; Wang, Q.; Lu, J.; Liu, C. T.; Yang, Y., High-entropy alloy: challenges and prospects. Materials Today 2016, 19 (6), 349-362.

23. Koo, W.-T.; Millstone, J. E.; Weiss, P. S.; Kim, I.-D., The Design and Science of Polyelemental Nanoparticles. ACS Nano 2020, 14 (6), 6407-6413.

24. George, E. P.; Raabe, D.; Ritchie, R. O., High-entropy alloys. Nature Reviews Materials 2019, 4 (8), 515-534.

25. Oses, C.; Toher, C.; Curtarolo, S., High-entropy ceramics. Nature Reviews Materials 2020, 5 (4), 295-309.

26. Rost, C. M.; Sachet, E.; Borman, T.; Moballegh, A.; Dickey, E. C.; Hou, D.; Jones, J. L.; Curtarolo, S.; Maria, J.-P., Entropy-stabilized oxides. Nature communications 2015, 6 (1), 1-8.

27. Zhou, J.; Zhang, J.; Zhang, F.; Niu, B.; Lei, L.; Wang, W., High-entropy carbide: a novel class of multicomponent ceramics. Ceramics International 2018, 44 (17), 22014-22018.

28. Wang, F.; Yan, X.; Wang, T.; Wu, Y.; Shao, L.; Nastasi, M.; Lu, Y.; Cui, B., Irradiation damage in $\left(\mathrm{Zr}_{0.25} \mathrm{Ta}_{0.25} \mathrm{Nb}_{0.25} \mathrm{Ti}_{0.25}\right) \mathrm{C}$ high-entropy carbide ceramics. Acta Materialia 2020, 195, 739-749.

29. Gild, J.; Zhang, Y.; Harrington, T.; Jiang, S.; Hu, T.; Quinn, M. C.; Mellor, W. M.; Zhou, N.; Vecchio, K.; Luo, J., High-Entropy Metal Diborides: A New Class of High-Entropy Materials and a New Type of Ultrahigh Temperature Ceramics. Scientific Reports 2016, 6 (1), 37946.

30. Castle, E.; Csanádi, T.; Grasso, S.; Dusza, J.; Reece, M., Processing and properties of highentropy ultra-high temperature carbides. Scientific reports 2018, 8 (1), 1-12.

31. Sarker, P.; Harrington, T.; Toher, C.; Oses, C.; Samiee, M.; Maria, J.-P.; Brenner, D. W.; Vecchio, K. S.; Curtarolo, S., High-entropy high-hardness metal carbides discovered by entropy descriptors. Nature Communications 2018, 9 (1), 4980.

32. Feng, L.; Chen, W.-T.; Fahrenholtz, W. G.; Hilmas, G. E., Strength of single-phase high-entropy carbide ceramics up to $2300^{\circ}$ C. Journal of the American Ceramic Society 2021, 104 (1), 419-427.

33. Wang, K.; Chen, L.; Xu, C.; Zhang, W.; Liu, Z.; Wang, Y.; Ouyang, J.; Zhang, X.; Fu, Y.; Zhou, Y., Microstructure and mechanical properties of (TiZrNbTaMo) C high-entropy ceramic. Journal of Materials Science \& Technology 2020, 39, 99-105.

34. Liu, D.; Zhang, A.; Jia, J.; Meng, J.; Su, B., Phase evolution and properties of (VNbTaMoW) C high entropy carbide prepared by reaction synthesis. Journal of the European Ceramic Society 2020, 40 (8), 2746-2751.

35. Gild, J.; Kaufmann, K.; Vecchio, K.; Luo, J., Reactive flash spark plasma sintering of high-entropy ultrahigh temperature ceramics. Scripta Materialia 2019, 170, 106-110.

36. Ye, B.; Wen, T.; Huang, K.; Wang, C.-Z.; Chu, Y., First-principles study, fabrication, and characterization of $\left(\mathrm{Hf}_{0.2} \mathrm{Zr}_{0.2} \mathrm{Ta}_{0.2} \mathrm{Nb}_{0.2} \mathrm{Ti}_{0.2}\right) \mathrm{C}$ high-entropy ceramic. Journal of the American Ceramic Society 2019, 102 (7), 4344-4352.

37. Wei, X.-F.; Liu, J.-X.; Li, F.; Qin, Y.; Liang, Y.-C.; Zhang, G.-J., High entropy carbide ceramics from different starting materials. Journal of the European Ceramic Society 2019, 39 (10), 2989-2994.

38. Ye, B.; Wen, T.; Liu, D.; Chu, Y., Oxidation behavior of $\left(\mathrm{Hf}_{0.2} \mathrm{Zr}_{0.2} \mathrm{Ta}_{0.2} \mathrm{Nb}_{0.2} \mathrm{Ti}_{0.2}\right) \mathrm{C}$ high-entropy ceramics at 1073-1473 K in air. Corrosion Science 2019, 153.

39. Tan, Y.; Chen, C.; Li, S.; Han, X.; Xue, J.; Liu, T.; Zhou, X.; Zhang, H., Oxidation behaviours of high-entropy transition metal carbides in $1200^{\circ} \mathrm{C}$ water vapor. Journal of Alloys and Compounds 2020, $816,152523$. 
40. Vladescu, A.; Titorencu, I.; Dekhtyar, Y.; Jinga, V.; Pruna, V.; Balaceanu, M.; Dinu, M.; Pana, I.; Vendina, V.; Braic, M., In vitro biocompatibility of Si alloyed multi-principal element carbide coatings. PLoS one 2016, 11 (8), e0161151.

41. Andreyev, A.; Kartmazov, G.; Chikryzhov, A.; Karpets, M.; Dolomanov, A.; Ostroverkh, A.; Kantsyr, E., Production and mechanical properties of high-entropic carbide based on the TiZrHfVNbTa multicomponent alloy. Journal of Superhard Materials 2017, 39 (3), 166-171.

42. Braic, V.; Balaceanu, M.; Braic, M.; Vladescu, A.; Panseri, S.; Russo, A., Characterization of multi-principal-element (TiZrNbHfTa)N and (TiZrNbHfTa)C coatings for biomedical applications. Journal of the Mechanical Behavior of Biomedical Materials 2012, 10, 197-205.

43. Hlova, I. Z.; Dolotko, O.; Boote, B. W.; Pathak, A. K.; Smith, E. A.; Pecharsky, V. K.; Balema, V. P., Multi-principal element transition metal dichalcogenides via reactive fusion of 3D-heterostructures. Chemical Communications 2018, 54 (89), 12574-12577.

44. Tian Lan, I. H., Vitalij Pecharsky, Arjun Pathak, Viktor Balema, and Sonal Padalkar, High-Entropy Transition Metal Dichalcogenides As Exceptional Electrocatalysts of Hydrogen Evolution Reaction (HER). ECS Meeting Abstracts 2019.

45. Blöchl, P. E., Projector augmented-wave method. Physical Review B 1994, 50 (24), 17953-17979.

46. Kresse, G.; Joubert, D., From ultrasoft pseudopotentials to the projector augmented-wave method. Physical Review B 1999, 59 (3), 1758-1775.

47. Dahlqvist, M.; Rosen, J., Predictive theoretical screening of phase stability for chemical order and disorder in quaternary 312 and 413 MAX phases. Nanoscale 2020, 12 (2), 785-794.

48. Pinto, D.; Anasori, B.; Avireddy, H.; Shuck, C. E.; Hantanasirisakul, K.; Deysher, G.; Morante, J. R.; Porzio, W.; Alshareef, H. N.; Gogotsi, Y., Synthesis and electrochemical properties of 2D molybdenum vanadium carbides-solid solution MXenes. Journal of Materials Chemistry A 2020, 8 (18), 8957-8968.

49. Sokol, M.; Natu, V.; Kota, S.; Barsoum, M. W., On the Chemical Diversity of the MAX Phases. Trends in Chemistry 2019, 1 (2), 210-223.

50. Naguib, M.; Bentzel, G. W.; Shah, J.; Halim, J.; Caspi, E. N.; Lu, J.; Hultman, L.; Barsoum, M. W., New Solid Solution MAX Phases: $\left(\mathrm{Ti}_{0.5}, \mathrm{~V}_{0.5}\right)_{3} \mathrm{AlC}_{2},\left(\mathrm{Nb}_{0.5}, \mathrm{~V}_{0.5}\right)_{2} \mathrm{AlC},\left(\mathrm{Nb}_{0.5}, \mathrm{~V}_{0.5}\right)_{4} \mathrm{AlC}_{3}$ and $\left(\mathrm{Nb}_{0.8}\right.$, $\left.\mathrm{Zr}_{0.2}\right)_{2} \mathrm{AlC}$. Materials Research Letters 2014, 2 (4), 233-240.

51. Yang, J.; Naguib, M.; Ghidiu, M.; Pan, L. M.; Gu, J.; Nanda, J.; Halim, J.; Gogotsi, Y.; Barsoum, M. W., Two-Dimensional Nb-Based $\mathrm{M}_{4} \mathrm{C}_{3}$ Solid Solutions (MXenes). Journal of the American Ceramic Society 2016, 99 (2), 660-666.

52. Alhabeb, M.; Maleski, K.; Anasori, B.; Lelyukh, P.; Clark, L.; Sin, S.; Gogotsi, Y., Guidelines for Synthesis and Processing of 2D Titanium Carbide $\left(\mathrm{Ti}_{3} \mathrm{C}_{2} \mathrm{~T}_{\mathrm{x}} \mathrm{MXene}\right)$. Chemistry of Materials 2017, 29 (18), 7633-7644.

53. Anasori, B.; Shi, C.; Moon, E. J.; Xie, Y.; Voigt, C. A.; Kent, P. R. C.; May, S. J.; Billinge, S. J. L.; Barsoum, M. W.; Gogotsi, Y., Control of electronic properties of 2D carbides (MXenes) by manipulating their transition metal layers. Nanoscale Horizons 2016, 1 (3), 227-234.

54. Hantanasirisakul, K.; Alhabeb, M.; Lipatov, A.; Maleski, K.; Anasori, B.; Salles, P.; Ieosakulrat, C.; Pakawatpanurut, P.; Sinitskii, A.; May, S. J.; Gogotsi, Y., Effects of Synthesis and Processing on Optoelectronic Properties of Titanium Carbonitride MXene. Chemistry of Materials 2019, 31, 2941-2951.

55. Fredrickson, K. D.; Anasori, B.; Seh, Z. W.; Gogotsi, Y.; Vojvodic, A., Effects of Applied Potential and Water Intercalation on the Surface Chemistry of $\mathrm{Ti}_{2} \mathrm{C}$ and $\mathrm{Mo}_{2} \mathrm{C}$ MXenes. The Journal of Physical Chemistry C 2016, 120 (50), 28432-28440.

56. Wyatt, B. C.; Rosenkranz, A.; Anasori, B., 2D MXenes: Tunable Mechanical and Tribological Properties. Advanced Materials 2021, 2007973.

57. Shekhirev, M.; Shuck, C. E.; Sarycheva, A.; Gogotsi, Y., Characterization of MXenes at every step, from their precursors to single flakes and assembled films. Progress in Materials Science 2020, 100757. 
58. Anasori, B.; Dahlqvist, M.; Halim, J.; Moon, E. J.; Lu, J.; Hosler, B. C.; El'ad, N. C.; May, S. J.; Hultman, L.; Eklund, P.; Rosen, J.; Barsoum, M. W., Experimental and theoretical characterization of ordered MAX phases $\mathrm{Mo}_{2} \mathrm{TiAlC}_{2}$ and $\mathrm{Mo}_{2} \mathrm{Ti}_{2} \mathrm{AlC}_{3}$. Journal of Applied Physics 2015, 118 (9), 094304.

59. Anasori, B.; Halim, J.; Lu, J.; Voigt, C. A.; Hultman, L.; Barsoum, M. W., Mo2 TiAlC 2 : A new ordered layered ternary carbide. Scripta Materialia 2015, 101, 5-7.

60. Meshkian, R.; Tao, Q.; Dahlqvist, M.; Lu, J.; Hultman, L.; Rosen, J., Theoretical stability and materials synthesis of a chemically ordered MAX phase, $\mathrm{Mo}_{2} \mathrm{ScAlC}_{2}$, and its two-dimensional derivate $\mathrm{Mo}_{2} \mathrm{ScC}_{2}$ MXene. Acta Materialia 2017, 125, 476-480.

61. Caspi, E. N.; Chartier, P.; Porcher, F.; Damay, F.; Cabioc'h, T., Ordering of (Cr,V) Layers in Nanolamellar $\left(\mathrm{Cr}_{0.5} \mathrm{~V}_{0.5}\right)_{\mathrm{n}+1} \mathrm{AlC}_{\mathrm{n}}$ Compounds. Materials Reseach Letters 2014, 1-7.

62. Liu, Z.; Zheng, L.; Sun, L.; Qian, Y.; Wang, J.; Li, M., $\left(\mathrm{Cr}_{2 / 3} \mathrm{Ti}_{1 / 3}\right)_{3} \mathrm{AlC}_{2}$ and $\left(\mathrm{Cr}_{5 / 8} \mathrm{Ti}_{3 / 8}\right)_{4} \mathrm{AlC}_{3}$ : New MAX-phase Compounds in Ti-Cr-Al-C System. Journal of the American Ceramic Society 2014, 97 (1), 67-69.

63. Dahlqvist, M.; Rosen, J., Order and disorder in quaternary atomic laminates from first-principles calculations. Physical Chemistry Chemical Physics 2015, 17 (47), 31810-31821.

64. Sang, X.; Xie, Y.; Lin, M.-W.; Alhabeb, M.; Van Aken, K. L.; Gogotsi, Y.; Kent, P. R. C.; Xiao, K.; Unocic, R. R., Atomic Defects in Monolayer Titanium Carbide $\left(\mathrm{Ti}_{3} \mathrm{C}_{2} \mathrm{~T}_{\mathrm{x}}\right)$ MXene. ACS Nano 2016, 10, 9193-9200.

65. Halim, J.; Cook, K. M.; Eklund, P.; Rosen, J.; Barsoum, M. W., XPS of cold pressed multilayered and freestanding delaminated 2D thin films of $\mathrm{Mo}_{2} \mathrm{TiC}_{2} \mathrm{~T}_{\mathrm{z}}$ and $\mathrm{Mo}_{2} \mathrm{Ti}_{2} \mathrm{C}_{3} \mathrm{~T}_{\mathrm{z}}$ (MXenes). Applied Surface Science 2019, 494, 1138-1147.

66. Chastain, J.; King Jr, R. C., Handbook of X-ray photoelectron spectroscopy. Perkin-Elmer Corporation 1992, 40, 221.

67. Halim, J.; Cook, K. M.; Naguib, M.; Eklund, P.; Gogotsi, Y.; Rosen, J.; Barsoum, M. W., X-ray photoelectron spectroscopy of select multi-layered transition metal carbides (MXenes). Applied Surface Science 2016, 362, 406-417.

68. Dahlqvist, M.; Alling, B.; Rosén, J., Stability trends of MAX phases from first principles. Physical Review B 2010, 81 (22), 220102.

69. Swalin, R. A.; Arents, J., Thermodynamics of solids. Journal of The Electrochemical Society 1962, 109 (12), 308C.

70. Dong, L.; Kumar, H.; Anasori, B.; Gogotsi, Y.; Shenoy, V. B., Rational Design of TwoDimensional Metallic and Semiconducting Spintronic Materials Based on Ordered Double-TransitionMetal MXenes. The Journal of Physical Chemistry Letters 2017, 8 (2), 422-428.

71. He, J.; Ding, G.; Zhong, C.; Li, S.; Li, D.; Zhang, G., $\mathrm{Cr}_{2} \mathrm{TiC}_{2}$-based double MXenes: novel 2D bipolar antiferromagnetic semiconductor with gate-controllable spin orientation toward antiferromagnetic spintronics. Nanoscale 2019, 11 (1), 356-364.

72. Yang, C.; Jin, S. Z.; Liang, B. Y.; Jia, S. S., Low-temperature synthesis of high-purity $\mathrm{Ti}_{3} \mathrm{AlC}_{2}$ by MA-SPS technique. Journal of the European Ceramic Society 2009, 29 (1), 181-185.

73. Barsoum, M. W., MAX Phases: Properties of Machinable Ternary Carbides and Nitrides. John Wiley \& Sons: 2013.

74. $\quad$ Van de Walle, A.; Tiwary, P.; De Jong, M.; Olmsted, D.; Asta, M.; Dick, A.; Shin, D.; Wang, Y.; Chen, L.-Q.; Liu, Z.-K., Efficient stochastic generation of special quasirandom structures. Calphad 2013, 42, 13-18.

75. Perdew, J. P.; Burke, K.; Ernzerhof, M., Generalized gradient approximation made simple. Physical review letters 1996, 77 (18), 3865.

76. Kresse, G.; Hafner, J., Ab initio molecular dynamics for liquid metals. Physical Review B 1993, 47 (1), 558.

77. Kresse, G.; Furthmüller, J., Efficiency of ab-initio total energy calculations for metals and semiconductors using a plane-wave basis set. Computational materials science 1996, 6 (1), 15-50.

78. Kresse, G.; Furthmüller, J., Efficient iterative schemes for ab initio total-energy calculations using a plane-wave basis set. Physical review B 1996, 54 (16), 11169. 
79. Monkhorst, H. J.; Pack, J. D., Special points for Brillouin-zone integrations. Physical review B 1976, 13 (12), 5188.

80. Manna, S.; Gorai, P.; Brennecka, G. L.; Ciobanu, C. V.; Stevanović, V., Large piezoelectric response of van der Waals layered solids. Journal of Materials Chemistry C 2018, 6 (41), 11035-11044. 